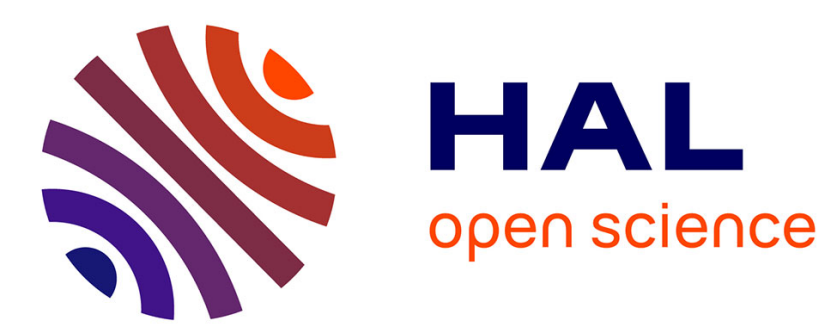

\title{
Lunar perturbation of the metric associated to the averaged orbital transfer
}

Bernard Bonnard, Helen Henninger, Jérémy Rouot

\section{To cite this version:}

Bernard Bonnard, Helen Henninger, Jérémy Rouot. Lunar perturbation of the metric associated to the averaged orbital transfer. Analysis and geometry in control theory and its applications, pp.65-84, 2015, 10.1007/978-3-319-06917-3_3 . hal-01090977v3

\section{HAL Id: hal-01090977 https://hal.inria.fr/hal-01090977v3}

Submitted on 17 Apr 2015

HAL is a multi-disciplinary open access archive for the deposit and dissemination of scientific research documents, whether they are published or not. The documents may come from teaching and research institutions in France or abroad, or from public or private research centers.
L'archive ouverte pluridisciplinaire HAL, est destinée au dépôt et à la diffusion de documents scientifiques de niveau recherche, publiés ou non, émanant des établissements d'enseignement et de recherche français ou étrangers, des laboratoires publics ou privés. 


\title{
Lunar perturbation of the metric associated to the averaged orbital transfer
}

\author{
Bernard Bonnard, Helen Henninger and Jérémy Rouot
}

\author{
Dedicated to Hélène Frankowska and Héctor Sussmann
}

\begin{abstract}
In a series of previous article [1,2, we introduced a Riemannian metric associated to the energy minimizing orbital transfer with low propulsion. The aim of this article is to study the deformation of this metric due to a standard perturbation in space mechanics, the lunar attraction. Using Hamiltonian formalism, we describe the effects of the perturbation on the orbital transfers and the deformation of the conjugate and cut loci of the original metric.
\end{abstract}

Keywords. Orbital transfer, optimal control, averaging methods.

\section{Introduction}

Recent space missions like lunar Smart-1 mission, Boeing orbital transfer, using electric propulsion are innovative design feature to reduce launch costs and lead to the analyse of the low thrust controlled Kepler equation using averaging techniques in optimal control. Pioneering work in this direction associated to the energy minimization problem are due to Edelbaum 8,9, Epenoy-Geffroy [10,11] and more recently to Bonnard-Caillau [1,2]. Under some simplifying assumption they lead to the definition of a Riemannian distance between Keplerian orbits, and this is a preliminary step in computing the time minimal or find mass maximizing solutions using numerical continuation techniques [6].

The objective of this article is to analyse the deformation of this metric taking into account the lunar perturbation which affect a wide range of missions. Again in the framework of the continuation techniques, we shall make simplifying assumptions. The main point is to deduce from the averaged system the qualitative policy to make the transfer and to initialize the shooting algorithm.

Work supported in part by the French Space Agency CNES, R\&T action R-S13/BS-005012 and by the region Provence-Alpes-Côte d'Azur. 
Making such assumptions leads to the analysis of a Zermelo navigation problem defined by an Hamiltonian which is a deformation of the Hamiltonian associated to the Riemannian metric and complete analysis of the transfer is made using continuation about trajectories computations and conjugate and cut analysis.

The organization of this article is the following. In section 2, we recall the computations and properties of the Riemannian metric based on 1 . In section 3 , we present lunar perturbation and we describe the averaged system. In section 4, we give numerical simulations computations on extremal trajectories and on conjugate loci. In section 5, we conclude by considering more general perturbations.

\section{The Riemannian metric}

The controlled Kepler equation, assuming the mass constant can be normalized to

$$
\frac{d^{2} q}{d t^{2}}=-\frac{q}{|q|^{3}}+u
$$

where $q=\left(q_{1}, q_{2}, q_{3}\right)$ is the position of the satellite and the thrust is bounded by $|u| \leq \epsilon$. The thrust can be decomposed in a moving frame $u=u_{1} F_{1}+$ $u_{2} F_{2}+u_{3} F_{3}$ e.g. the so-called radial-orthoradial frame: $F_{1}=\frac{q}{|q|}, F_{2}=F_{3} \wedge F_{1}$ and $F_{3}=\frac{q \wedge \dot{q}}{|q \wedge \dot{q}|}$. The state of the system is described by an angle: the true longitude $l$ and by five equinoctial elements $x$ corresponding to first integrals of the uncontrolled motion. For instance, $x=(P, e, h)$ where $P$ is the semilatus rectum of the osculating conic, $e=\left(e_{x}, e_{y}\right)$ is the eccentricity vector and $h=\left(h_{x}, h_{y}\right)$ is the inclination vector. We restrict the system to the elliptic domain, that is to the manifold $\mathcal{X}$ of elliptic trajectories of the Kepler equation $\mathcal{X}=\{P>0,|e|<1\}$.

The system takes the form

$$
\begin{aligned}
\frac{d x}{d t} & =\sum_{i=1}^{3} u_{i} F_{i}(x, l) \\
\frac{d l}{d t} & =w_{0}(x, l)+g(x, l, u) .
\end{aligned}
$$

An important problem is to transfer the satellite between coplanar orbits, the corresponding subsystem is deduced by setting both the inclination $h$ and the control $u_{3}$ to zero.

The energy minimization problem is studied in detail in [1] and we present only the main results.

The control is rescaled using $u=\epsilon v,|v| \leq 1$ to introduce the small parameter $\epsilon$ and we consider the energy minimization problem to transfer the system from $\left(x_{0}, l_{0}\right)$ to a terminal orbit $x_{F}$. The terminal cumulated longitude is also fixed to $l_{F}$. Parametrizing the trajectory by the cumulated 
longitude $l$, the system is written

$$
\frac{d x}{d l}=\frac{\epsilon}{w_{0}(x, l)+\epsilon g(x, l, u)} \sum_{i=1}^{3} v_{i} F_{i}(x, l)
$$

and the cost function to minimize is

$$
\epsilon^{2} \int_{l_{0}}^{l_{F}} \frac{|v|^{2} d l}{w_{0}(x, l)+\epsilon g(x, l, u)} .
$$

In order to perform the analytic computation, we first relax the bound $|v| \leq 1$. Indeed, for a fixed $\epsilon$, the constraint will be fulfilled by a big enough final longitude $l_{F}$.

Using the maximum principle [15], optimal trajectories are extremals, integral curves of the following Hamiltonian

$$
H_{\epsilon}(x, l, p, v)=\frac{\epsilon}{w_{0}(x, l)+\epsilon g(x, l, u)}\left(p^{0} \epsilon|v|^{2}+\sum_{i=1}^{3} v_{i} P_{i}\right)
$$

where $p^{0} \leq 0$ and $P_{i}=\left\langle p, F_{i}\right\rangle, i=1,2,3$. By controllability properties of the system we can restrict to the normal case $p^{0}<0$ and it can be normalized to $-\frac{1}{2 \epsilon}$. As a result, up to first order $\epsilon$, we have the approximation

$$
H_{\epsilon}(x, l, p, v)=\frac{\epsilon}{w_{0}(x, l)}\left(-\frac{1}{2}|v|^{2}+\sum_{i=1}^{3} v_{i} P_{i}\right)+o(\epsilon) .
$$

In the computation of the averaged system, we can used the first order approximation

$$
H(x, l, p, v)=\frac{1}{2} \sum_{i=1}^{3}\left(\frac{P_{i}}{\sqrt{w_{0}}}\right)^{2}
$$

since the trajectories are $C^{0}$-closed [1].

Definition 2.1. The averaged Hamiltonian is

$$
\langle H\rangle(x, p)=\frac{1}{2 \pi} \int_{0}^{2 \pi} H(x, l, p) d l .
$$

Coplanar case. We have $H=\frac{1}{2 \omega_{0}} \sum_{i=1}^{2} P_{i}^{2}$ and the averaged system is expressed in the coordinates $(n, \rho, \theta)$ where $n=a^{-3 / 2}$ is the mean movement, $a$ is the semi-major axis, $\rho$ is the eccentricity and $\theta$ is the polar angle of the vector $\left(e_{x}, e_{y}\right)$ (hence $\left.\rho=\sqrt{e_{x}^{2}+e_{y}^{2}}\right)$,

$$
P=\frac{1-\rho^{2}}{n^{\frac{2}{3}}}, e_{x}=\rho \cos (\theta), e_{y}=\rho \sin (\theta),
$$

and we have

Proposition 2.2. In coordinates $(n, \rho, \theta)$, the averaged Hamiltonian is

$$
\left\langle H_{1}\right\rangle=\frac{1}{4 n^{\frac{5}{3}}}\left[18 n^{2} p_{n}^{2}+5\left(1-\rho^{2}\right) p_{\rho}^{2}+\left(5-4 \rho^{2}\right) \frac{p_{\theta}^{2}}{\rho^{2}}\right]
$$


and $\left\langle H_{1}\right\rangle$ is the Hamiltonian of the Riemannian metric

$$
d s^{2}=\frac{1}{9 n^{\frac{1}{3}}} d n^{2}+\frac{2 n^{\frac{5}{3}}}{5\left(1-\rho^{2}\right)} d \rho^{2}+\frac{2 n^{\frac{5}{3}}}{5-4 \rho^{2}} \rho^{2} d \theta^{2} .
$$

The coordinates $(n, \rho, \theta)$ are orthogonal coordinates.

Non Coplanar Case. The complete Hamiltonian is $H=\frac{1}{2}\left(P_{1}^{2}+P_{2}^{2}+P_{3}^{2}\right)$. As previously we use $(n, \rho, \theta)$ as coordinates and we make a polar representation of $h$,

$$
h_{x}=\sigma \cos (\Omega), h_{y}=\sigma \sin (\Omega)
$$

where the angle $\Omega$ is the longitude of the ascending node and $\sigma=\sin \left(\frac{i}{2}\right)$. Introducing $\theta=\theta-\Omega$, the angle of the pericenter, and denoting

$$
p_{\theta \Omega}=\frac{2 \sigma^{2}}{\sigma^{2}+1} p_{\theta}+p_{\Omega},
$$

we have

Proposition 2.3. The averaged Hamiltonian of the non-coplanar transfer is

$$
\langle H\rangle=\left\langle H_{1}\right\rangle+\left\langle H_{2}\right\rangle
$$

with

$\left\langle H_{2}\right\rangle=\frac{\left(\sigma^{2}+1\right)^{2}}{16 n^{\frac{5}{3}}} \times\left[\frac{1+4 \rho^{2}}{1-\rho^{2}}\left(\cos (\theta) p_{\sigma}+\sin (\theta) \frac{p_{\theta \Omega}}{\sigma}\right)^{2}+\left(-\sin (\theta) p_{\sigma}+\cos (\theta) \frac{p_{\theta \Omega}}{\sigma}\right)^{2}\right]$ and $\langle H\rangle$ is associated with a five-dimensional Riemannian metric.

\section{Properties of the metric (coplanar case).}

1. The metric associated to $\left\langle H_{1}\right\rangle$

$$
g=\frac{2}{9 n^{\frac{1}{3}}} d n^{2}+\frac{4 n^{\frac{5}{3}}}{5\left(1-\rho^{2}\right)} d \rho^{2}+\frac{4 n^{\frac{5}{3}}}{5-4 \rho^{2}} \rho^{2} d \theta^{2}
$$

is isomorphic to $g=d r^{2}+r^{2}\left(d \phi^{2}+G(\phi) d \theta^{2}\right)$ where $r=\frac{2^{3 / 2}}{5} n^{\frac{5}{6}}$, $\phi=\frac{1}{c} \arcsin (\rho), G(\phi)=\frac{25}{2} \frac{\sin ^{2}(c \phi)}{1+4 \cos ^{2}(c \phi)}$ and $c=\sqrt{\frac{2}{5}}$.

2. The metric $g$ is Liouville integrable with a linear first integral and the geodesic flow can be integrated using elementary functions.

The perturbed case. The system is written

$$
\frac{d x}{d l}=P\left(x, l, l^{\prime}\right)+\sum_{i=1}^{3} u_{i} F_{i}(x, l)
$$

where $P$ is the perturbation associated to the lunar perturbation, depending on an additional angular variable $l^{\prime}$, e.g. the lunar longitude or the mean anomaly. The averaging procedure will produce an Hamiltonian which is the superposition of

- An averaged perturbation denoted $\left\langle H_{P}\right\rangle$.

- The averaged Hamiltonian $\langle H\rangle$ computed before and corresponding to the minimization problem. 
This leads to the definition of a Zermelo navigation problem 3, 4].

Definition 2.4. A Zermelo navigation problem on a n-dimensional Riemannian manifold $(\mathcal{X}, g)$ is a time minimal problem associated to the system

$$
\frac{d x}{d l}=F_{0}(x)+\sum_{i=1}^{n} u_{i} F_{i}(x)
$$

where $F_{i}$ form an orthonormal frame for the metric $g$ and $|u| \leq 1$. Observe that $F_{0}$ represents the current of magnitude $\left|F_{0}\right|_{g}$. If $\left|F_{0}\right|_{g}<1$, this defines a Finsler metric.

Definition 2.5. If we apply the Maximum principle to the previous optimal problem this defines an Hamiltonian which is homogeneous in $p$. Conversely, one can associate to the Hamiltonian $H=\left\langle H_{P}\right\rangle+\lambda \sqrt{\langle H\rangle}$ a Zermelo navigation problem, where $\lambda$ is a scaling parameter associated to the maximal control magnitude.

\section{The perturbations}

\subsection{Preliminairies}

First of all, the perturbations lead to the definition of a vector field whose trajectories behavior can be roughly classified in the framework of properties of conservative systems in the large, introduced for the three-body problem by Poincaré [14] and see [12 for a modern presentation.

Definition 3.1. Let $V$ be a smooth complete vector field on a manifold $\mathcal{M}$ and let $x\left(t, x_{0}\right)$ be the solution starting at $t=0$ from $x_{0}$. The point $x_{0}$ is called Poisson-stable if for every neighbourhood $U$ of $x_{0}$ and every $T \geq 0$, there exists $t_{1}, t_{2} \geq T$ such that $x\left(t_{1}, x_{0}\right)$ and $x\left(-t_{2}, x_{0}\right)$ belong to $U$. The point $x_{0}$ is said to be departing if for each compact set $K$ there exists $T \geq 0$ such that if $|t| \geq T, x\left(t, x_{0}\right) \notin K$.

Theorem 3.2. Let $V$ be smooth complete conservative vector field on $(\mathcal{M}, \theta)$, then almost every point is Poisson-stable or departing.

Hence Poisson stability corresponds to bounded motions. A more precise description was recently deduced from KAM theory [5] which is briefly presented below.

Definition 3.3. A solution of the system

$$
\left\{\begin{array}{l}
\dot{I}=0 \\
\dot{\phi}=w(I)
\end{array}\right.
$$

where $(I, \phi)$ are variables in $\left(\mathbb{R}^{d}, \mathbb{T}^{d}\right)$ is called quasi-periodic.

Proposition 3.4. There exists quasi-periodic solutions in the restricted circular planar three body problem. 
Coordinates. The satellite position and velocity are represented by $(q, \dot{q})$ and we denote $(q, p)$ the standard symplectic coordinates. The motion of the satellite which is defined up to a proper normalization by Kepler Hamiltonian

$$
H(q, p)=\frac{1}{2}|p|^{2}-\frac{1}{|q|} .
$$

To analyze the effect of a perturbing force deriving from a potential $R$, one uses the Lagrange equations 13,17$]$.

$$
\begin{array}{lr}
\frac{d a}{d t}= & \frac{1}{n^{2} a} \frac{\partial R}{\partial \tau} \\
\frac{d \rho}{d t} & \frac{1-\rho^{2}}{n^{2} a^{2}} \frac{\partial R}{\partial \tau}-\frac{\sqrt{1-\rho^{2}}}{n a^{2} \rho} \frac{\partial R}{\partial \theta} \\
\frac{d i}{d t}= & \frac{\cot (i)}{n a^{2} \sqrt{1-\rho^{2}}} \frac{\partial R}{\partial \theta}-\frac{1}{n a^{2} \sin (i) \sqrt{1-\rho^{2}}} \frac{\partial R}{\partial \Omega} \\
\frac{d \Omega}{d t}= & \frac{1}{n a^{2} \sin (i) \sqrt{1-\rho^{2}}} \frac{\partial R}{\partial i} \\
\frac{d \theta}{d t}= & \frac{\sqrt{1-\rho^{2}}}{n a^{2} \rho} \frac{\partial R}{\partial \rho}-\frac{\cot (i)}{n a^{2} \sqrt{1-\rho^{2}}} \frac{\partial R}{\partial i} \\
\frac{d \tau}{d t}= & \frac{2}{n^{2} a} \frac{\partial R}{\partial a}+\frac{1-\rho^{2}}{n^{2} a^{2} \rho} \frac{\partial R}{\partial \rho}
\end{array}
$$

where $i$ is the angle of inclination between the orbital plane of the satellite and the orbital plane of the Moon, $\Omega$ is the longitude of the ascending node, $\theta$ is the angle of the perigee and $\tau$ is the time of the perigee passage.

Remark. It is useful to introduce the mean anomaly $M$ to locate the satellite on its orbit. It is defined by the relation $M=n(t-\tau)$. In this case, we set $R(a, e, i, \Omega, \omega, \tau)=\tilde{R}(a, e, i, \Omega, \omega, M)$ and the partial derivatives verify

$$
\frac{\partial R}{\partial \tau}=-n \frac{\partial \tilde{R}}{\partial M}, \quad \frac{\partial R}{\partial a}=\frac{\partial \tilde{R}}{\partial a}+\frac{n a}{2} \frac{d n}{d t}(t-\tau) .
$$

The effect of the lunar perturbation on the satellite motions are well understood and we use the computations excerpted from [13]. They are related to solar perturbation of the Moon. In this reference, they study Moon motion under the Sun perturbation, which we can adapt to the Earth-Moon-satellite case.

\subsection{The lunar perturbation}

Given a geocentric inertial frame of reference, the perturbing lunar potential can be expressed by

$$
R\left(q, q^{\prime}\right)=\tilde{\mu}^{\prime}\left(\frac{1}{\left|q-q^{\prime}\right|}-\frac{q \cdot q^{\prime}}{r^{\prime 3}}\right)
$$


where $\tilde{\mu}^{\prime}=\mu^{\prime} / m_{\text {Earth }}$ is the standard gravitational parameter of the Moon divide by the mass of the Earth, $q$ (resp. $q^{\prime}$ ) is the position vector of the satellite (resp. the Moon) and $|q|$ (resp. $\left.\left|q^{\prime}\right|\right)$ is denoted by $r$ (resp. $r^{\prime}$ ). The potential (3) stands for the dynamics of the satellite of the two body problem Earth-satellite which is perturbed by the Moon.

Setting the reference plane as the orbital plane of the Moon, $q^{\prime}$ can be decomposed in terms of the osculating elements $\left(x^{\prime}=\left(n^{\prime}, \rho^{\prime}, \theta^{\prime}\right), M^{\prime}\right)$ of the Moon where $n^{\prime}=\tilde{\mu}^{\prime} / a^{\prime 3 / 2}$ is the mean movement, $a^{\prime}$ the semi-major axis, $\rho^{\prime}$ is the eccentricity, $\theta^{\prime}$ the angle of the perigee and $M^{\prime}$ the mean anomaly.

In order to have a rough evaluation of the perturbation, we use a simplified model in 13. based on the following assumptions : the eccentricity of the satellite $\rho$ is small and the inclination $i$ of the satellite with respect to the Moon orbital plane is small.

We have,

$$
\frac{1}{\left|q-q^{\prime}\right|}=\frac{1}{r^{\prime} \sqrt{1+\left(\frac{r}{r^{\prime}}\right)^{2}-2 \frac{r}{r^{\prime}} \cos (\Psi)}}
$$

where $\Psi$ is the angle between the two vectors $q$ and $q^{\prime}$.

Assume the satellite on a low Earth orbit, then $r \ll r^{\prime}$ and using Legendre polynomials $P_{k}$

$$
\frac{1}{\left|q-q^{\prime}\right|}=\frac{1}{a^{\prime}} \sum_{k=0}^{\infty} \alpha^{k}\left(\frac{r}{a}\right)^{k}\left(\frac{a^{\prime}}{r^{\prime}}\right)^{k+1} P_{k}(\cos (\Psi))
$$

where $\alpha=\frac{a}{a^{\prime}} \ll 1$.

Using the approximation

$$
\frac{1}{\left|q-q^{\prime}\right|}=\frac{r}{r^{\prime 2}} \cos (\Psi)+\frac{1}{a^{\prime}}\left[1+\frac{1}{2} \alpha^{2}\left(\frac{r}{a}\right)^{2}\left(\frac{a^{\prime}}{r^{\prime}}\right)^{3}\left(-1+3 \cos ^{2}(\Psi)\right)\right]+o\left(\alpha^{3}\right) .
$$

The perturbing potential expression becomes

$$
\begin{aligned}
R & =\frac{\tilde{\mu}^{\prime}}{2 a^{\prime}}\left(\alpha^{2}\left(\frac{r}{a}\right)^{2}\left(\frac{a^{\prime}}{r^{\prime}}\right)^{3}\left(-1+3 \cos ^{2}(\Psi)\right)\right)+o\left(\alpha^{3}\right) \\
& =\frac{n^{\prime 2}}{2 n^{4 / 3}}\left(\frac{r}{a}\right)^{2}\left(\frac{a^{\prime}}{r^{\prime}}\right)^{3}\left(-1+3 \cos ^{2}(\Psi)\right)+o\left(\alpha^{3}\right) .
\end{aligned}
$$

where the first term $\frac{1}{a^{\prime}}$ in 4 has been removed since it does not depend on the satellite orbital elements $(x=(n, e, i, \theta, \Omega), M)$.

\section{Development of the terms $\frac{r}{a}$ and $\frac{a^{\prime}}{r^{\prime}}$}

From the Kepler's equation the eccentric anomaly $E$ satisfies $E=M+$ $\rho \sin (E)$, the $2 \pi$-periodic function $E \mapsto \rho \sin (E)$ can be expanded into Fourier series and $E$ can be expressed as

$$
E=M+\sum_{k=1}^{\infty} a_{k} \sin (k M)
$$


where $a_{k}=\frac{2}{\pi} \int_{0}^{\pi} \rho \sin (E) \sin (k M) d M=\frac{2}{k} J_{k}(k \rho)$, denoting $J_{m}(z)$ the Bessel functions of the first kind defined as

$$
J_{m}(z)=\frac{1}{\pi} \int_{0}^{\pi} \cos (m \theta-z \sin (\theta)) d \theta,(m \in \mathbb{Z}, z \in \mathbb{C}) .
$$

Therefore,

$$
E=M+\sum_{k=1}^{\infty} \frac{2}{k} J_{k}(k \rho) \sin (k M)
$$

With a similar method (see for instance [16]), one obtains that

$$
\cos (E)=-\frac{e}{2}+\sum_{k=1}^{\infty} \frac{2}{k^{2}} \frac{d}{d \rho}\left[J_{k}(k \rho)\right] \cos (k M) .
$$

From the relations,

$$
\begin{aligned}
& \frac{a}{r}=\frac{1}{n} \dot{E} \\
& \frac{r}{a}=1-\rho \cos (E),
\end{aligned}
$$

we have the following expansions,

$$
\begin{aligned}
& \frac{a^{\prime}}{r^{\prime}}=1+2 \sum_{k=1}^{\infty} J_{k}(k \rho) \cos (k M), \\
& \frac{r}{a}=1+\frac{\rho^{2}}{2}-\sum_{k=1}^{\infty} \frac{2 \rho}{k^{2}} \frac{d}{d \rho}\left[J_{k}(k \rho)\right] \cos (k M) .
\end{aligned}
$$

In the sequel we use Maple software in the computations.

\section{Development of the term $\cos ^{2}(\Psi)$}

The spherical trigonometry allows us to express the angle $\Psi$ in terms of the orbital elements of the satellite and the Moon

$$
\cos (\Psi)=\cos (\theta+v) \cos \left(\theta^{\prime}+v^{\prime}-\Omega\right)+\cos (i) \sin (\theta+v) \sin \left(\theta^{\prime}+v^{\prime}-\Omega\right)
$$

where $v$ and $v^{\prime}$ are respectively the true anomaly of the satellite and the Moon.

We have

$$
\cos (v)=\frac{\cos (E)-\rho}{1-\rho \cos (E)}, \quad \sin (v)=\frac{\sqrt{1-\rho^{2}} \sin (E)}{1-\rho \cos (E)} .
$$

Hence

$$
R\left(x, M, x^{\prime}, M^{\prime}\right)=\sum_{k, m \in \mathbb{Z}} C_{k, m}\left(x, x^{\prime}\right) \exp \left(I\left(k M+m M^{\prime}\right)\right)
$$

and more precisely

$$
R\left(x, M, x^{\prime}, M^{\prime}\right)=\sum_{k \in \mathbb{Z}^{5}} \tilde{C}_{k}\left(a, \rho, i, a^{\prime}, \rho^{\prime}\right) \exp (I\langle k, \zeta\rangle) .
$$


where $\zeta=\left(\Omega, \theta, M, \theta^{\prime}, M^{\prime}\right)$.

Computations lead to

$$
\begin{aligned}
& R\left(x, M, x^{\prime}, M^{\prime}\right)=\frac{n^{\prime 2}}{n^{4 / 3}}\left[1 / 4\left(1+3 / 2 \rho^{2}+3 / 2 \rho^{\prime 2}-6 \sin ^{2}(i / 2)\right)\right. \\
& -1 / 2 \rho \cos (M)+3 / 4 \rho^{\prime} \cos \left(M^{\prime}\right)-1 / 8 \rho^{2} \cos (2 M) \\
& +9 / 8 \rho^{\prime 2} \cos \left(2 M^{\prime}\right)-3 / 4 \rho \rho^{\prime} \cos \left(M-M^{\prime}\right) \\
& -3 / 4 \rho \rho^{\prime} \cos \left(M+M^{\prime}\right)+3 / 2 \sin ^{2}(i / 2) \cos (2 \theta+2 M) \\
& +3 / 2 \sin ^{2}(i / 2) \cos \left(2 \theta^{\prime}+2 M^{\prime}-2 \Omega\right) \\
& +\frac{15}{8} \rho^{2} \cos \left(-2 \Omega-2 \theta+2 \theta^{\prime}+2 M^{\prime}\right) \\
& -\frac{63}{8} \rho \rho^{\prime} \cos \left(2 \theta+2 \Omega+M-2 \theta^{\prime}-3 M^{\prime}\right) \\
& -9 / 4 \rho \cos \left(2 \theta+2 \Omega+M-2 \theta^{\prime}-2 M^{\prime}\right) \\
& +9 / 8 \rho \rho^{\prime} \cos \left(2 \theta+2 \Omega+M-2 \theta^{\prime}-M^{\prime}\right) \\
& +\left(\frac{3}{4}-\frac{3}{2} \sin ^{2}(i / 2)-\frac{15}{8} \rho^{\prime 2}-\frac{15}{8} \rho^{2}\right) \cos \left(2 \theta+2 \Omega+2 M-2 \theta^{\prime}-2 M^{\prime}\right) \\
& +\frac{21}{8} \rho \rho^{\prime} \cos \left(2 \theta+2 \Omega+3 M-2 \theta^{\prime}-3 M^{\prime}\right) \\
& +3 / 4 \rho \cos \left(2 \theta+2 \Omega+3 M-2 \theta^{\prime}-2 M^{\prime}\right) \\
& -3 / 8 \rho \rho^{\prime} \cos \left(2 \theta+2 \Omega+3 M-2 \theta^{\prime}-M^{\prime}\right) \\
& +3 / 4 \rho^{2} \cos \left(2 \theta+2 \Omega+4 M-2 \theta^{\prime}-2 M^{\prime}\right) \\
& +\frac{51}{8} \rho^{\prime 2} \cos \left(-2 \theta^{\prime}-4 M^{\prime}+2 \theta+2 \Omega+2 M\right) \\
& +\frac{21}{8} \rho^{\prime} \cos \left(-2 \theta^{\prime}-3 M^{\prime}+2 \theta+2 \Omega+2 M\right) \\
& \left.-3 / 8 \rho^{\prime} \cos \left(-2 \theta^{\prime}-M^{\prime}+2 \theta+2 \Omega+2 M\right)\right]+o(\alpha)^{3}+o(\rho)^{3}+o\left(\rho^{\prime}\right)^{3} \text {. }
\end{aligned}
$$

Definition 3.5. The double averaged potential is defined by

$$
\langle\langle R\rangle\rangle_{M, M^{\prime}}\left(x, x^{\prime}\right)=\frac{1}{(2 \pi)^{2}} \int_{0}^{2 \pi} R\left(x, M, x^{\prime}, M^{\prime}\right) d M d M^{\prime} .
$$

Then, the double averaged of the potential (5) is

$$
\langle\langle R\rangle\rangle_{M, M^{\prime}}\left(x, x^{\prime}\right)=\frac{n^{\prime 2}}{4 n^{4 / 3}}\left(1+3 / 2 \rho^{2}+3 / 2 \rho^{\prime 2}-6 \sin ^{2}(i / 2)\right)
$$

(see 7] for more precise expansions). A simplified academic model is to set the eccentricity $\rho^{\prime}$ to zero, the inclination $i$ to zero and to restrict the control to this plane, the lunar perturbing potential becomes

$$
\langle\langle R\rangle\rangle_{M, M^{\prime}}\left(x, x^{\prime}\right)=\frac{n^{\prime 2}}{4 n^{4 / 3}}\left(1+\frac{3}{2} \rho^{2}\right) .
$$




\section{Computations}

\subsection{Shooting equation}

Let $\vec{H}$ be an Hamiltonian vector field associated to the Zermelo navigation problem, $z=(x, p), x \in \mathcal{X}$, denoting the state and adjoint vector and $H$ being homogeneous of degree 1 in $p$. Fixing the initial and final state vectors $\left(x_{0}, x_{1}\right)$ and $t_{F}$ being the transfer time the shooting equation is defined by

$$
S: p_{0} \mapsto \Pi\left(\exp \left(t_{F} \vec{H}\left(z_{0}\right)\right)=x_{1}\right.
$$

where $z_{0}=\left(x_{0}, p_{0}\right), \Pi:(x, p) \mapsto x$ and $p_{0}$ can be normalized by homogeneity.

\subsection{The geometric concept of conjugate point}

Definition 4.1. Let $z=(x, p)$ be a reference extremal solution of $\vec{H}$ on $\left[0, t_{F}\right]$. The variational equation

$$
\dot{\delta} z(t)=d \vec{H}(z(t)) \delta z(t)
$$

is called the Jacobi equation. A Jacobi field is a non trivial solution $\delta z=$ $(\delta x, \delta p)$ of Jacobi equation and it is said to be vertical at time $t$ if $\delta x(t)=0$.

Definition 4.2. We define the exponential mapping

$$
\exp _{x_{0}, t}\left(p_{0}\right)=\Pi\left(z\left(t, x_{0}, p_{0}\right)\right)
$$

where $p_{0}$ can be restricted to the sphere $\left|p_{0}\right|=1$. If $z=(x, p)$ is the reference extremal, a time $t_{c}>0$ is said to be conjugate to 0 if the mapping $p_{0} \mapsto$ $\exp _{x_{0}, t}\left(p_{0}\right)$ is not of rank $n-1$ at $t=t_{c}$ (with $n=\operatorname{dim} \mathcal{X}$ ) and the associated point $x\left(t_{c}\right)$ is said to be conjugate to $x_{0}$. We denote by $t_{1 c}$ the first conjugate time and $C\left(x_{0}\right)$ is the conjugate locus formed by the set of first conjugate points.

Testing conjugary. An algorithm can be deduced which is implemented in the Hampath Code [6] used in our numerical simulations. Let $z(t)=(x(t), p(t))$ be the reference extremal and consider the vector space of dimension $n-1$ generated by the Jacobi fields $\delta z_{i}=\left(\delta x_{i}, \delta p_{i}\right), i=1, \ldots, n-1$ vertical at $t=0$ and such that $\delta p_{i}(0)$ is orthogonal to $p_{0}$. At a conjugate time $t_{c}$, one has

$$
\operatorname{rank}\left[\delta x_{1}\left(t_{c}\right), \ldots, \delta x_{n-1}\left(t_{c}\right)\right]<n-1
$$

or equivalently,

$$
\operatorname{det}\left[\delta x_{1}\left(t_{c}\right), \ldots, \delta x_{n-1}\left(t_{c}\right), \dot{x}\left(t_{c}\right)\right]=0 .
$$

Hampath Code. This code is used to

- Integrate the Hamiltonian flow and compute the Jacobi fields along a given solution.

. Solve the shooting equation. 


\subsection{Computations}

We are in the coplanar case and Lagrange equations (2) give using (6)

$$
\begin{aligned}
& \frac{d n}{d t}=\frac{d \rho}{d t}=0 \\
& \frac{d \theta}{d t}=\frac{n^{1 / 3} \sqrt{1-\rho^{2}}}{\rho}\left[\frac{3 n^{\prime 2} \rho}{4 n^{4 / 3}}\right]=\frac{3 n^{\prime 2} \sqrt{1-\rho^{2}}}{4 n} .
\end{aligned}
$$

This leads to the averaged Hamiltonian

$$
\langle H\rangle(n, \rho, \theta)=p_{\theta}\left[\frac{3 n^{\prime 2} \sqrt{1-\rho^{2}}}{4 n}\right]+\lambda \sqrt{\frac{1}{4 n^{5 / 3}}\left[18 n^{2} p_{n}^{2}+5\left(1-\rho^{2}\right) p_{\rho}^{2}+\left(5-4 \rho^{2}\right) \frac{p_{\theta}^{2}}{\rho^{2}}\right]}
$$

where $\lambda$ is the scaling parameter of the control maximal magnitude.

\subsection{Numerical results}

This section is achieved by a series of numerical computations on the free system, on extremal trajectories and on conjugate loci. The simulations are computed thanks to the Hampath code [6].

Free system. The control is set to zero and the dynamical system is deduced from the Lagrange equations and the perturbative potential (6). The solutions are expressed in the $(n(t), \psi(t), \theta(t))$ coordinates where $\phi$ is the angle such that $\rho=\sin (\phi)$, and $\psi(t)=\frac{\pi}{2}-\phi(t)$.

Figures 1 and 2 yield two first integrals of the double averaged Hamiltonian system. The double average is taken with respect to the mean motion variables $M$ and $M^{\prime}$ which correspond respectively to the satellite and the Moon. This integral over $\left(M, M^{\prime}\right) \in[0,2 \pi] \times[0,2 \pi]$ is computed with $M^{\prime}$ fixed with respect to $M$ and the slow variables.

The variation of $\theta(t)$ is equal to $\frac{3}{4} \frac{n^{\prime 2}}{n}\left(1-\frac{\rho^{2}}{2}\right) \approx 2.8^{\circ}$ during one lunar revolution around the Earth.

Controlled system. Due to the homogeneity of order 1 of the Hamiltonian (7), the time-minimum problem is considered and the adjoint vector is normalized. Extremal trajectories are computed for different values of $\lambda$. The shooting algorithm is performed to solve the boundary value problem by determining the initial adjoint vector $p(0)$ and the optimal time $t_{f}$.

In the following figures, the perturbed case $\left(\lambda=10^{-1}\right)$ is represented in dashdot line and is compared to the unperturbed case represented in solid line. The final points are indicated by cross markers. The first conjugate points, indicated by star markers, are computed thanks to the algorithm presented in the subsection 4.2 for which the time evolution of the determinant of the matrix $\left(\delta x_{1}(t) \delta x_{2}(t) \dot{x}(t)\right)$ is presented in Figure 8 .

Figures 9 and 10 represent the projection of the extremal trajectories in $(\psi, \theta)$ coordinates starting from the initial point $\left(\rho_{0}, \theta_{0}\right)=(0.60, \pi)$ in the unperturbed and perturbed case. 

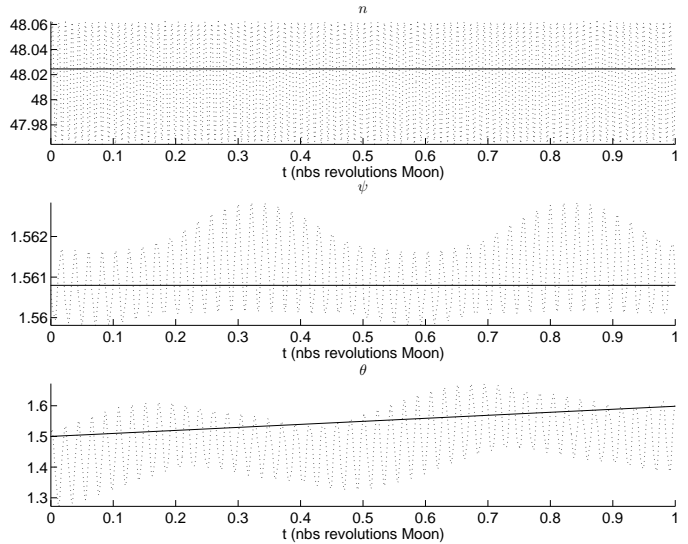

Figure 1. Evolution of $n, \psi$ and $\theta$ of the double averaged (solid line) and the non averaged (dotted line) free system over one lunar revolution around the Earth.
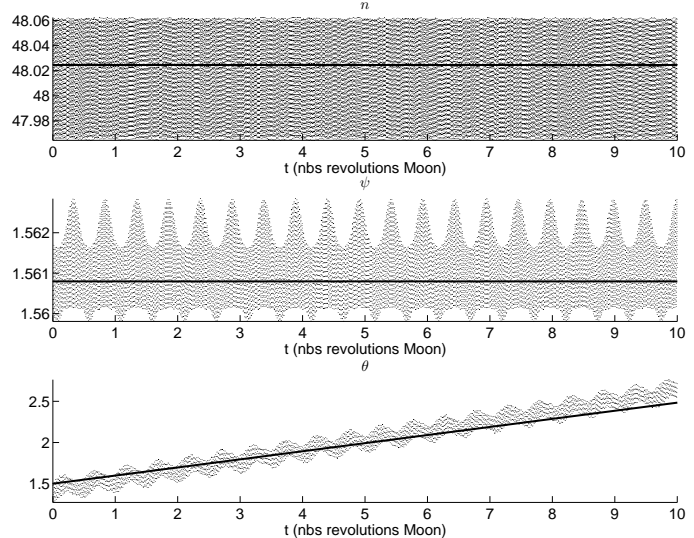

Figure 2. Evolution of $n, \psi$ and $\theta$ of the double averaged (solid line) and the non averaged (dotted line) free system over ten lunar revolutions around the Earth.

\section{Conclusion}

More general perturbations can be considered such as the $\mathrm{J}_{2}$-effect.

\subsection{A brief description of the $\mathbf{J}_{2}$-effect 13}

The Earth is modelled by an homogeneous oblate ellipsoid of revolution whose axis of symmetry is identified to the axis of rotation passing through the pole denoted $O z$ and the position of the satellite can be represented in spherical coordinates $(r, \lambda, \phi), \lambda$ being the latitude and $\phi$ the longitude. 

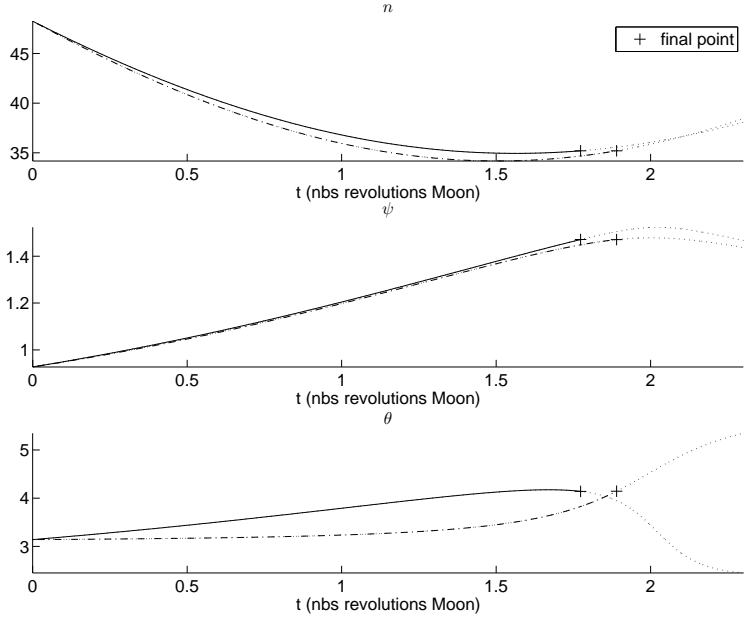

FiguRE 3. Evolution of state vectors of extremal trajectories from the initial state point $\left(n_{0}, \rho_{0}, \theta_{0}\right)=(48.3,0.60, \pi)$ to the final state point $\left(n_{f}, \rho_{f}, \theta_{f}\right)=(35.2,0.10, \pi+1)$. The comparison is performed between the perturbed case (dashdot line) and the unperturbed one (solid line). Final points are indicated (cross markers).
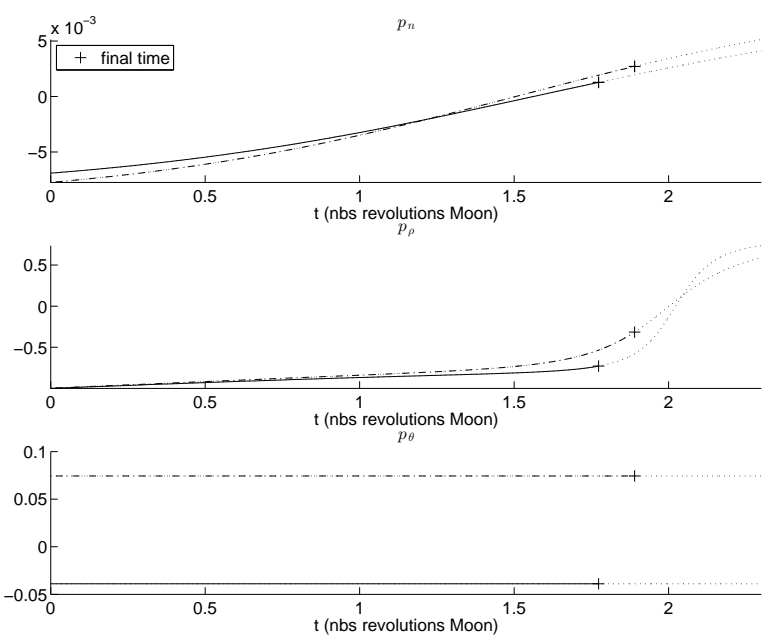

FiguRE 4. Evolution of adjoint vectors of extremal trajectories from the initial state point $\left(n_{0}, \rho_{0}, \theta_{0}\right)=(48.3,0.60, \pi)$ to the final state point $\left(n_{f}, \rho_{f}, \theta_{f}\right)=(35.2,0.10, \pi+1)$. Final points are indicated. 

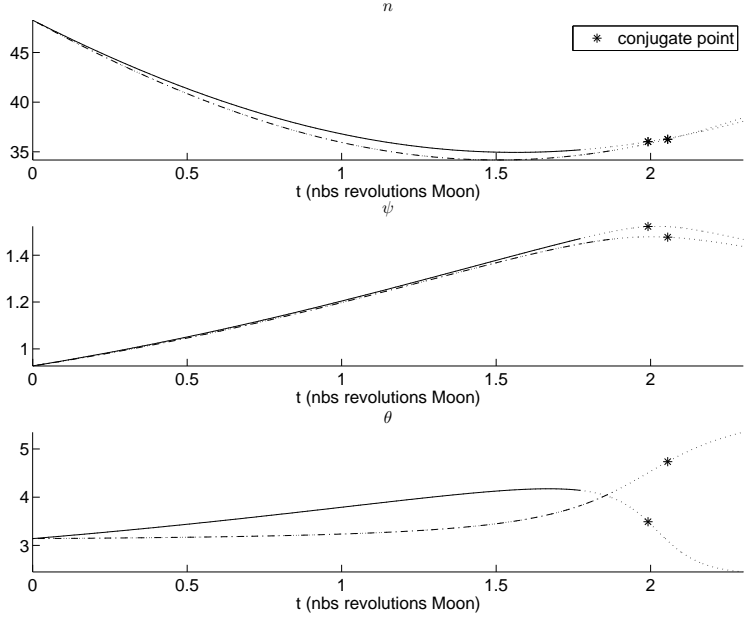

FiguRE 5. Evolution of state vectors of extremal trajectories from the initial state point $\left(n_{0}, \rho_{0}, \theta_{0}\right)=(48.3,0.60, \pi)$ to $\left(n_{f}, \rho_{f}, \theta_{f}\right)=(35.2,0.10, \pi+1)$. Conjugate points are indicated (star markers).
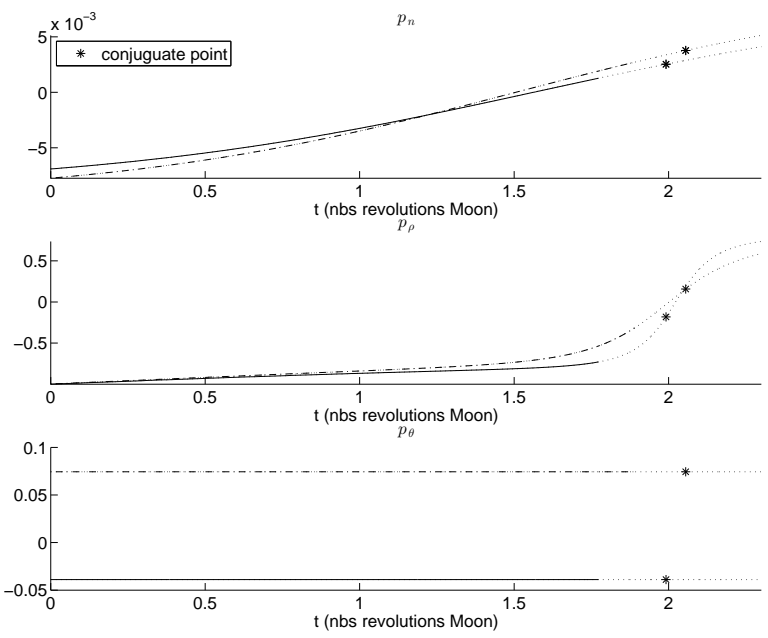

Figure 6. Evolution of adjoint vectors of extremal trajectories from the initial state point $\left(n_{0}, \rho_{0}, \theta_{0}\right)=(48.3,0.60, \pi)$ to the final state point $\left(n_{f}, \rho_{f}, \theta_{f}\right)=(35.2,0.10, \pi+1)$. Conjugate points are indicated.

The perturbing potential in the normalized coordinates takes the form

$$
R_{2}=\frac{1}{2 r}\left(\frac{R_{e}}{r}\right)^{2} J_{2}\left(1-3 \sin ^{2}(\phi)\right)
$$




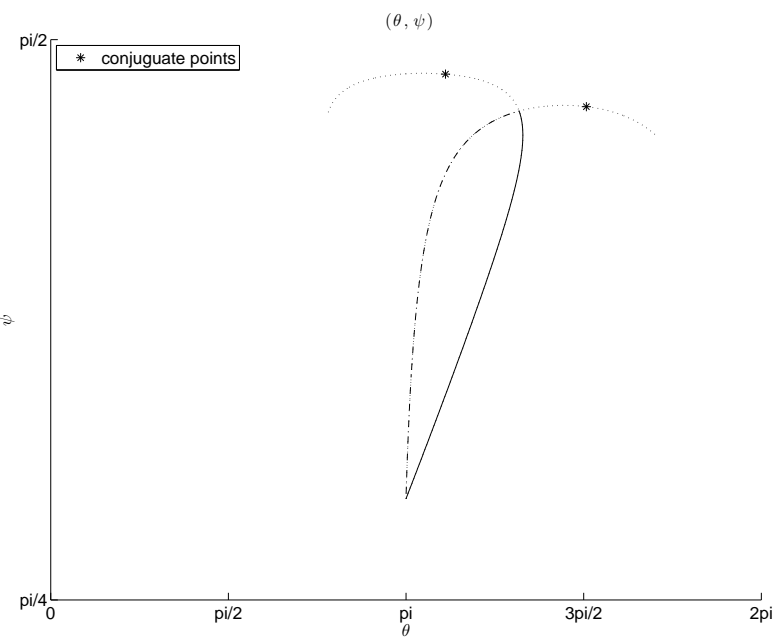

Figure 7. Projection of extremal trajectories in $(\psi, \theta)$ coordinates in the perturbed case (dash-dot line) and the unperturbed one (solid line).

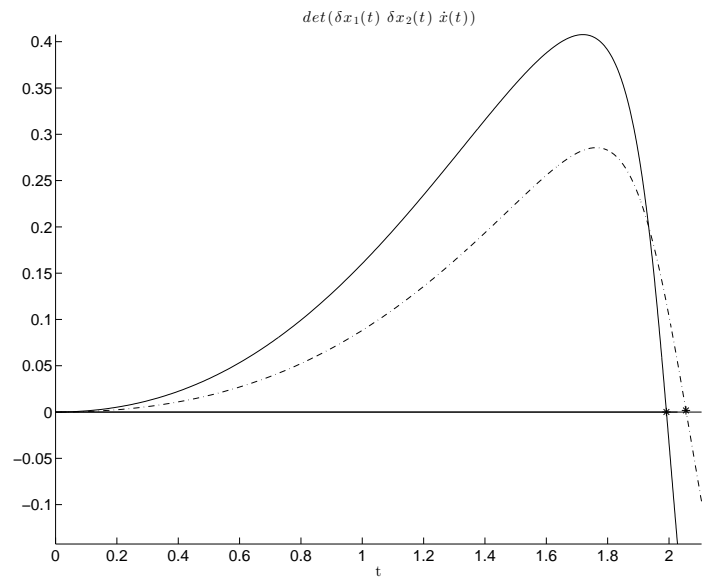

FiguRE 8. Rank condition for the determination of the first conjugate point for the perturbed case and the unpertubed one. The first zero of the determinant is the first conjugate time.

where we have the relation

$$
\sin (\phi)=\sin (i) \sin (\theta+v)
$$

where $v$ is the true anomaly. Hence the perturbing potential is given by

$$
R_{2}=\frac{3}{2} \frac{R_{e}^{2} J_{2}}{a^{3}}\left(\frac{a}{r}\right)^{3}\left[\frac{1}{3}-\frac{1}{2} \sin ^{2}(i)+\frac{1}{2} \sin ^{2}(i) \cos (2(\theta+v))\right]
$$




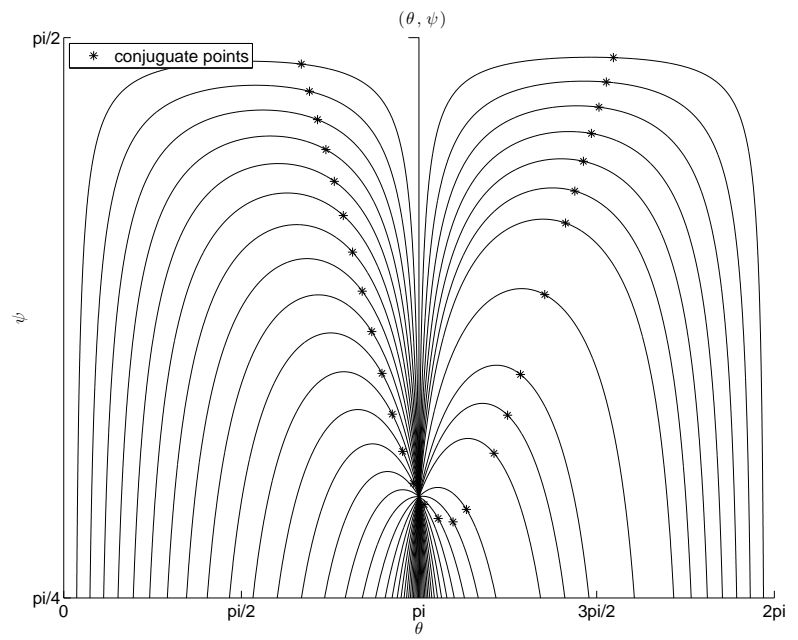

Figure 9. Projection of extremal trajectories in the unperturbed case in $(\psi, \theta)$ coordinates starting from the same initial point $\left(\rho_{0}, \theta_{0}\right)=(0.60, \pi)$. Conjugate points are indicated.

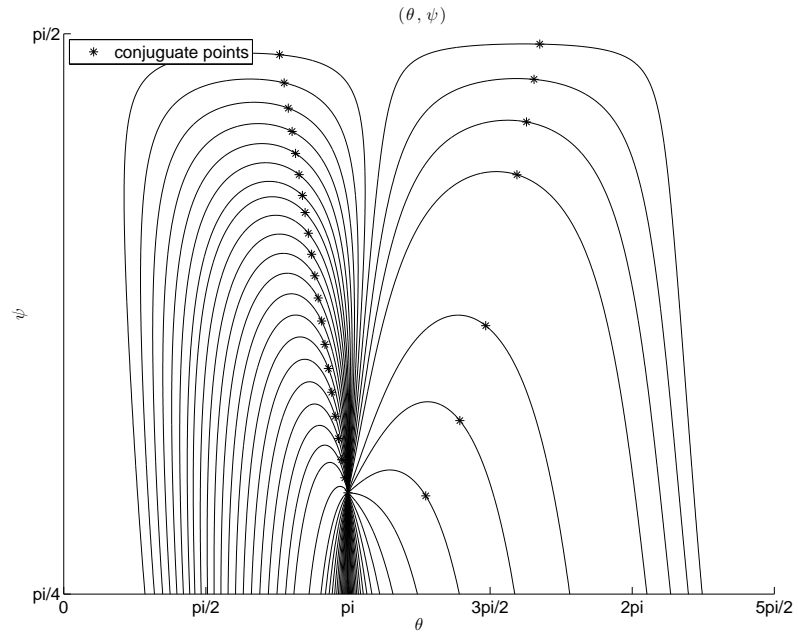

Figure 10. Projection of extremal trajectories in the perturbed case $(\lambda=1)$ in $(\psi, \theta)$ coordinates starting from the same initial point $\left(\rho_{0}, \theta_{0}\right)=(0.60, \pi)$. Conjugate points are indicated.

where $R_{e}$ is the mean Earth's equatorial radius and $J_{2}=1.08263 .10^{-3}$ is a constant. 
The averaged perturbation computed with the formula

$$
\left\langle R_{2}\right\rangle_{M}=\frac{1}{2 \pi} \int_{0}^{2 \pi} R d M
$$

where $M$ is the mean anomaly gives the following.

Proposition 5.1. The averaged perturbation associated to the $J_{2}$-effect is described by the potential

$$
\left\langle R_{2}\right\rangle_{M}=\frac{3}{2} \frac{R_{e}^{2} J_{2}}{a^{3}\left(1-\rho^{2}\right)^{\frac{3}{2}}}\left(\frac{1}{3}-\frac{1}{2} \sin ^{2}(i)\right) .
$$

\subsection{Additional perturbations 17}

In practise one may encounter other perturbations : solar perturbations and non conservative type of perturbations such as atmosphere drag and solar eclipses.

\subsection{Extensions}

Similar computations as in [7] will lead to more accurate model useful with longer time transfers. Finally the lunar perturbation and the $\mathrm{J}_{2}$-effect can be superposed and their effects numerically analysed for space mission where both effects have to be taken into account.

\section{References}

[1] B. Bonnard and J.-B. Caillau. Riemannian metric of the averaged energy minimization problem in orbital transfer with low thrust. Ann. Inst. H. Poincaré Anal. Non Linéaire, 24(3):395-411, 2007.

[2] B. Bonnard and J.-B. Caillau. Geodesic flow of the averaged controlled Kepler equation. Forum Math., 21(5):797-814, 2009.

[3] Arthur E. Bryson, Jr. and Yu Chi Ho. Applied optimal control. Hemisphere Publishing Corp. Washington, D. C., 1975.

[4] C. Carathéodory. Calculus of variations and partial differential equations of the first order. Part I: Partial differential equations of the first order. Chelsea Publishing Compagny, New York, 1982.

[5] A. Celletti and L. Chierchia. KAM stability for a three-body problem of the solar system. Z. Angew. Math. Phys., 57(1):33-41, 2006.

[6] O. Cots. Contrôle optimal géométrique: méthodes homotopiques et applications. $\mathrm{PhD}$ thesis, Université de Bourgogne, 2012.

[7] R. C. Domingos, R. Vilhena de Moraes, and A. F. Bertachini De Almeida Prado. Third-body perturbation in the case of elliptic orbits for the disturbing body. Math. Probl. Eng., pages Art. ID 763654, 14, 2008.

[8] T. N. Edelbaum. Optimum low-thrust rendezvous and station keeping. AIAA J., 2:1196-1201, 1964.

[9] T. N. Edelbaum. Optimum power-limited orbit transfer in strong gravity fields. AIAA J., 3:921-925, 1965. 
[10] S. Geffroy. Généralisation des techniques de moyennation en contrôle optimal, application aux problèmes de rendez-vous orbitaux à poussée faible. $\mathrm{PhD}$ thesis, INPT, 1997.

[11] S. Geffroy and R. Epenoy. Optimal low-thrust transfers with constraintsgeneralization of averaging techniques. Acta Astronautica, 41(3):133-149, 1997.

[12] V. V. Nemytskii and V. V. Stepanov. Qualitative theory of differential equations. Princeton University Press, Princeton, N.J., 1960.

[13] G. Pascoli. Astronomie fondamentale. Dunod, Paris, 2000.

[14] H. Poincaré. Euvres. Tome VII. Éditions Jacques Gabay, Sceaux, 1996.

[15] L. S. Pontryagin, V. G. Boltyanskii, R. V. Gamkrelidze, and E. F. Mishchenko. The mathematical theory of optimal processes. Interscience Publishers John Wiley \& Sons, Inc. New York-London, 1962.

[16] J. P. Vinti, G. J. Der, and N. L. Bonavito. Orbital and celestial mechanics. Published by American Institute of Aeronautics and Astronautics, 1998.

[17] O. Zarrouati. Trajectoires spatiales. CEPADUES-EDITIONS, Toulouse, 1987.

Bernard Bonnard

Institut de Mathématiques de Bourgogne,

9 avenue Savary,

21078 Dijon, France.

e-mail: bernard.bonnard@u-bourgogne.fr

Helen Henninger

INRIA,

2004 route des lucioles,

F-06902 Sophia Antipolis, France.

e-mail: helen.henninger@inria.fr

Jérémy Rouot

INRIA,

2004 route des lucioles,

F-06902 Sophia Antipolis, France.

e-mail: jeremy.rouot@inria.fr 